\title{
Epigallocatechin Gallate-Stearate Enhances the Efficacy of Antibiotics
}

\author{
Ayuni Yussof ${ }^{1,2}$, Umme Habiba ${ }^{2}$, Deborah Liaw ${ }^{2}$, Tinchun Chu ${ }^{*}$, Lee H. Lee ${ }^{2 *}$ \\ ${ }^{1}$ Department of Biological Sciences, Seton Hall University, South Orange, NJ, USA \\ ${ }^{2}$ Department of Biology, Montclair State University, Montclair, NJ, USA \\ Email: *Tin-Chun.Chu@shu.edu, ^Lee.Lee@montclair.edu
}

How to cite this paper: Yussof, A., Habiba, U., Liaw, D., Chu, T. and Lee, L.H. (2019) Epigallocatechin Gallate-Stearate Enhances the Efficacy of Antibiotics. Open Journal of Medical Microbiology, 9, 77-94. https://doi.org/10.4236/ojmm.2019.93009

Received: July 8, 2019

Accepted: August 27, 2019

Published: August 30, 2019

Copyright $\odot 2019$ by author(s) and Scientific Research Publishing Inc. This work is licensed under the Creative Commons Attribution International License (CC BY 4.0).

http://creativecommons.org/licenses/by/4.0/

(c) (i) Open Access

\begin{abstract}
Introduction: The rise in antibiotic resistant cases has caused a global concern. Researchers around the world are trying to find a novel alternative to combat this issue. Green tea with its many health benefits, including antibacterial and antiviral activity, has shown to be one of the most promising candidates to be used as an agent to solve this problem. Objective: This study focuses on evaluating the synergistic effects of antibiotics and two green tea polyphenols: epigallocatechin gallate (EGCG), and its modified lipophilic form epigallocatechin gallate stearate (EGCG-S). Methods: In this study, twelve antibiotics and eight bacteria: Gram-positive Staphylococcus aureus ( $S$. aureus), Staphylococcus epidermidis (S. epidermidis) and Bacillus megaterium (B. megaterium); Gram-negative Escherichia coli (E. coli), Pseudomonas aeruginosa ( $P$. aeruginosa), Serratia marcescens ( $S$. marcescens), and Enterobacter aerogenes (E. aerogenes); and acid-fast Mycobacterium smegmatis (M. smegmatis) were used. Antibacterial synergism profiling of EGCG, EGCG-S and antibiotics has been established using a disk diffusion assay. Results: The results revealed that both 1\% of EGCG and 1\% EGCG-S enhanced the antimicrobial activities on antibiotics in various bacteria. Antimicrobial susceptibility study indicated that EGCG-S was able to enhance some antibiotics from the resistant category to intermediate or susceptible and/or from intermediate category to susceptible. Both EGCG and EGCG-S worked comparably on Gram-positive bacteria; in $S$. aureus, both compounds enhanced 5 antibiotics (AM10, CF30, C30, S10 and TE30) activities while EGCG-S had higher efficiency. B. megaterium were susceptible to most of the antibiotic treatment, thus the impact of EGCG and EGCG-S was insignificant. EGCG-S worked better than EGCG on Gram-negative bacteria; converted 9 antibiotics susceptibility in $E$. coli and $P$. aeruginosa, and 8 antibiotics in E. aerogenes. EGCG and EGCG-S also showed synergism on acid-fast bacteria $M$. smegmatis with EGCG-S has much higher efficiency than EGCG.
\end{abstract}


Conclusion: The results suggested that EGCG-S might be a promising antibacterial synergistic agent with antibiotics to combat antibiotic-resistant bacteria.

\section{Keywords}

EGCG, EGCG-Stearate, Antibiotic Resistance, Disk Diffusion Methods

\section{Introduction}

Antibiotics have been a key element of modern medicine, leading to their extended use in healthcare and agriculture [1]. The extensive global use of antibiotics, lack of new and effective antibiotics and increased spread of multi-drug resistant bacteria have led to the looming threat to global health [2]. This rapid accumulation of antibiotic resistance suggests a reservoir of transferable antibiotic resistance gene determinants in response to antibiotics that were found in hospitals, environmental isolates, and even in permafrost DNA [3]. Evidence of antibiotic resistance in hospitals suggests they may contribute to increasing rates of nosocomial infections or hospital-associated infections. A group of pathogens named "ESKAPE (Enterococcus faecium, Staphylococcus aureus, Klebsiella pneumoniae, Acinetobacter baumannii, Pseudomonas aeruginosa and Enterobacter species)" has been widely studied because they are the leading cause of nosocomial infections and are generally characterized by multi-drug resistance [4] [5]. With multi-drug resistance representing one of the top threats to public health globally, novel natural alternatives are tested to help combat the rise of antibiotic resistant bacteria.

Green tea extracted from Camellia sinensis has been shown to have a wide range of health benefits including anticarcinogenic, anti-atherogenic, antimicrobial and antiviral properties [6]. Green tea extract contains a wide range of tea polyphenols including epigallocatechin-gallate (EGCG), epigallocatechin (EGC), epicatechin gallate (ECG) and epicatechin (EC) [2]. EGCG is the most abundant polyphenol with the greatest antibacterial activities among the tea polyphenols [7]. One of the advantages of EGCG is that it is non-toxic and can be applied or consumed without adverse effects, which the Federal Drug Administration (FDA) classifies as a safe compound [8] [9]. Under normal conditions, EGCG is a water-soluble polyphenol oxidized and metabolized rapidly, which leads to the loss of potent antimicrobial abilities quickly [10]. To overcome the stability issue, the modified lipophilic polyphenols could be an effective agent as green tea polyphenols (GTPs) [11]. EGCG-stearate (EGCG-S), lipid soluble tea polyphenols (LTPs) that were prepared chemically and enzymatically, can significantly improve the bioavailability of GTPs [12] [13].

Gram-negative bacteria like Escherichia coli (E. coli), Enterobacter aerogenes (E. aerogenes), Pseudomonas aeruginosa ( $P$. aeruginosa), and Serratia marces- 
cens ( $S$. marcescens) antibiotic resistance reached critical level with limited treatment options [14]. Some of the commonly isolated gram-positive bacteria like $B a-$ cillus megaterium (B. megaterium), Staphylococcus aureus (S. aureus), and Staphylococcus epidermidis ( $S$. epidermidis) have been shown to be have an increase number of resistant cases [15]. EGCG has been shown to have an inhibitory effect on both Gram-positive and Gram-negative bacteria, but the exact mechanism has not been determined [16] [17] [18].

This study uses both EGCG and EGCG-S along with twelve different antibiotic disks to evaluate the effect of these polyphenols on antibiotics in eight different Gram+, Gram- and acid-fast bacteria. The combination of antibiotics with either EGCG or EGCG-S provides insight into whether the addition of either compound can produce a synergistic effect and enhance the antimicrobial activity by these antibiotics.

\section{Material and Methods}

\subsection{Culture Preparation and Maintenance}

Eight microorganisms were included in this study: Gram-positive, Gram-negative, and acid-fast. Gram-positive bacteria: Bacillus megaterium (B. megaterium), Staphylococcus aureus (S. aureus), and Staphylococcus epidermis (S. epidermidis); Gram-negative bacteria: Escherichia coli (E. coli), Pseudomonas aeruginosa ( $P$. aeruginosa), Serratia marcescens ( $S$. marcescens), and Enterobacter aerogenes (E. aerogenes); and acid-Fast bacteria: Mycobacterium smegmatis (M. smegmatis) (Carolina Biological Supply Co.). These bacteria were maintained in nutrient broth (NB) and nutrient agar (NA) plate (BD Difco). The media was prepared according to manufacturer directions.

An overnight culture of each bacterium was prepared for each experiment. The overnight culture was grown at $37^{\circ} \mathrm{C}$ with a constant shaking at $250 \mathrm{rpm}$ except for Serratia marcescens, which was grown at room temperature without any shaking. The overnight cultures were checked for purity before used in each experiment.

The stock culture was prepared and stored at $4^{\circ} \mathrm{C}$ and an original stock for each bacterium was stored at $30 \%$ glycerol at $-80^{\circ} \mathrm{C}$.

\subsection{EGCG and EGCG-S Solution Preparation}

Pure compound of EGCG and EGCG-S were purchased from Camellix, LLC (Augusta, GA). The $1 \%$ stock solutions were prepared by dissolving 1 gram of EGCG or EGCG-S in $100 \mathrm{ml}$ of $100 \%$ ethyl alcohol (200 proof), followed by a 0.45 $\mu \mathrm{m}$ membrane filter sterilization.

\subsection{Kirby-Bauer Disk Diffusion Assay}

Twelve antibiotics with or without 1\% EGCG or EGCG-S were evaluated by Kirby-Bauer assay: Ampicillin (AM10), Bacitracin (B10), Cephalothin (CF30), Chloramphenicol (C30), Doxycycline (D30), Erythromycin (E15), Gentamicin 
(GM10), Penicillin (P10), Polymyxin B (PB300), Rifampin (RA5), Streptomycin (S10), and Tetracycline (TE30). The plates were incubated at $37^{\circ} \mathrm{C}$ for 24 and 48 hours. The zone of inhibition (ZOI) was recorded and the categories, susceptible $(\mathrm{S})$, intermediate (I) or resistant (R), were determined based on the criteria published by the Clinical and Laboratory Standards Institute (CLSI) [19]. All experiments were carried out in triplicates and the mean and standard deviation were calculated. The percentage of inhibition was calculated based on the Equation (1) listed below:

$$
\text { Percentage of Inhibition }(\%)=[(A-B) / B] \times 100
$$

where $A$ is the ZOI of the combined treatment and $B$ is the ZOI of the antibiotic alone.

\section{Results}

\subsection{EGCG and EGCG-S Showed Synergy on Antibiotics in Three Gram-Positive Bacteria}

Three Gram-positive bacteria: B. megaterium, $S$. aureus and $S$. epidermidis, were used in this study. B. megaterium is a Gram-positive bacillus, and an endospore former. The $\%$ of increase and $\%$ of decrease were calculated using ZOI and shown in Figure 1(a). From the ZOI study, $B$. megaterium was generally susceptible to antibiotic treatments; 10 of 12 antibiotics work on this organism. $B$. megaterium was only intermediate to B10 and TE30. However, addition of EGCG-S converted B. megaterium from being intermediate to susceptible to B10 treatment. These results indicate that EGCG-S may be a synergistic agent in enhancing the antimicrobial activity of antibiotics. EGCG and EGCG-S increased the antibiotic efficacy ranging from $4.00 \%$ to $44.54 \%$ and $18.80 \%$ to $112.04 \%$ respectively (Figure 1(b)). C30 (44.54\%) and B10 (112.04\%) had the most significant increase for EGCG and EGCG-S respectively. EGCG had a positive impact on 9 antibiotics (AM10, B10, CF30, C30, E15, GM10, P10, RA5 and S10) and EGCG-S had a positive impact on 7 (AM10, B10, CF30, E15, P10, RA5 and S10) with antibiotics.

The results from disk diffusion test for $S$. aureus (Figure 2(a)) suggest that this bacterium is susceptible to 6 (B10, D30, E15, GM10, PB300, RA5) of 12 antibiotics; intermediate to 4 antibiotics (CF30, C30, S10 and TE30) and resistant to 2 antibiotics (AM10 and P10). Addition of EGCG and EGCG-S changed the category from intermediate to susceptible for all 4 antibiotics (CF30, C30, S10 and TE30). EGCG converted AM10 from resistant to intermediate and EGCG-S converted it to susceptible.

EGCG and EGCG-S increased the antibiotic efficacy ranging from $6.71 \%$ to $34.21 \%$ and $16.02 \%$ to $57.94 \%$ respectively (Figure $2(\mathrm{~b})$ ). S10 (34.21\%) and P10 (57.94\%) had the most significant increases for EGCG and EGCG-S respectively. Although EGCG-S had the largest positive impact on P10, it still could not convert P10 into a susceptible treatment for $\mathcal{S}$. aureus. 
The results from disk diffusion test for $S$. epidermidis suggest that this bacterium was susceptible to only three antibiotics (D30, GM10, and S10). It was intermediate to 7 antibiotics (AM10, B10, CF30, C30, PB300, RA5 and TE30) and

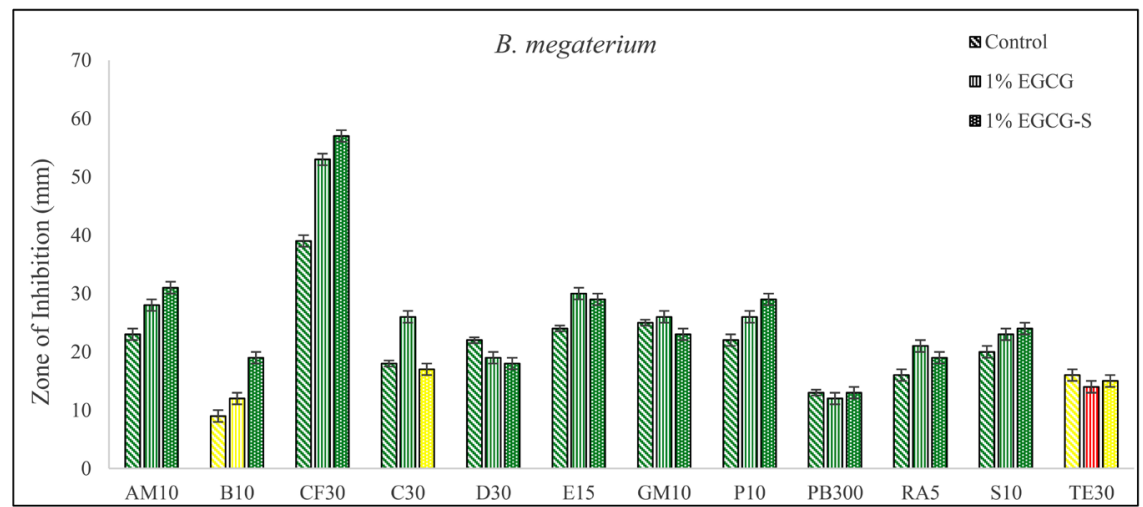

(a)

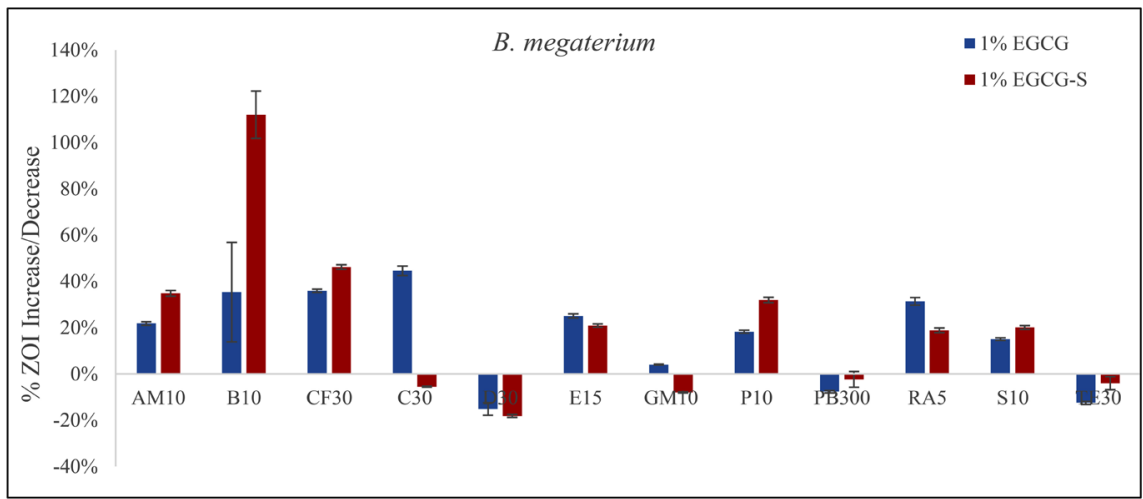

(b)

Figure 1. (a) The ZOI of B. megaterium when treated with $1 \%$ EGCG and $1 \%$ EGCG-S in combination with various antibiotics. The green color indicates susceptible, yellow indicates intermediate and red indicates resistant. The slanted stripe bar graph indicates antibiotic alone, the vertical stripe bar graph indicates antibiotic plus EGCG and the polka dot bar graph indicate antibiotic plus EGCG-S $(\mathrm{n}=3)$; (b) The percentage of ZOI increase/decrease of $B$. megaterium when treated with 1\% EGCG and 1\% EGCG-S in combination with various antibiotics. The blue bar represent antibiotic plus EGCG, and the red bar represent antibiotic plus EGCG-S $(n=3)$.

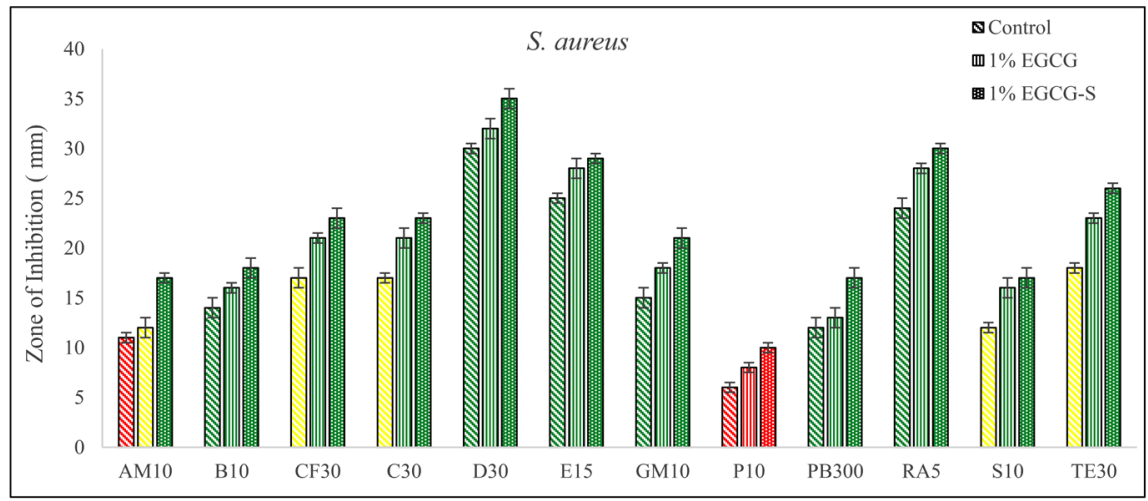

(a) 


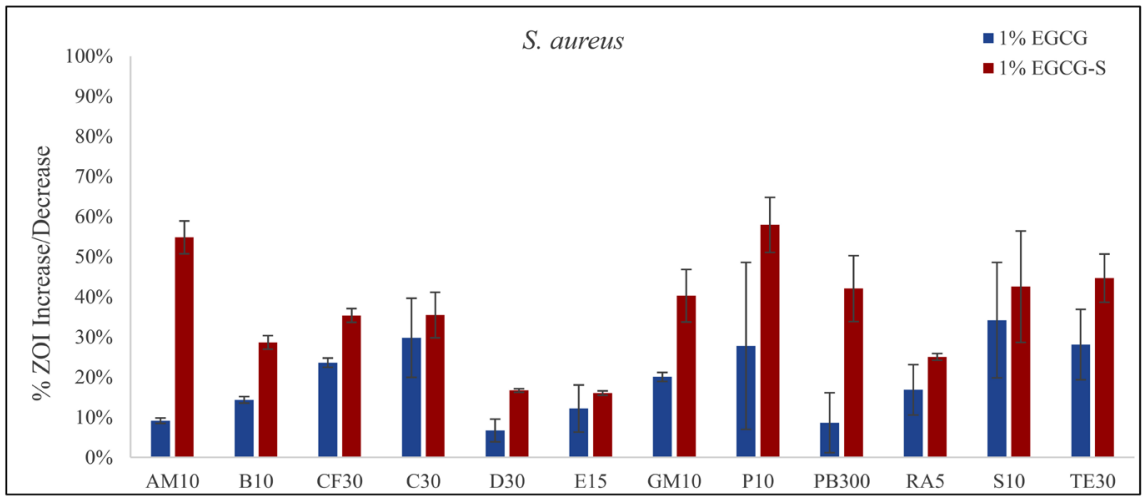

(b)

Figure 2. (a) The ZOI of $S$. aureus when treated with 1\% EGCG and 1\% EGCG-S in combination with antibiotics. The green color indicates susceptible, yellow indicates intermediate and red indicates resistant. The slanted stripe bar graph indicates antibiotic alone, the vertical stripe bar graph indicates antibiotic plus EGCG and the polka dot bar graph indicate antibiotic plus EGCG-S ( $\mathrm{n}=3$ ); (b) The percentage of ZOI increase/decrease of $S$. aureus when treated with 1\% EGCG and 1\% EGCG-S in combination with antibiotics. The blue bar represents antibiotic plus EGCG, and the red bar represents antibiotic plus EGCG-S $(\mathrm{n}=3)$.

was resistant to E15 and P10. With EGCG, the antibiotic profiles changed and $S$. epidermidis became susceptible to all of the antibiotics except E15 and PB300. EGCG-S changed most antibiotic profiles to susceptible with the exception of E15, PB300, and TE30. EGCG-S changed P10 to the intermediate category (Figure 3(a)). EGCG and EGCG-S increased the antibiotic efficacy ranging from $30.89 \%$ to $216.20 \%$ and $2.78 \%$ to $112.04 \%$ respectively. P10 had the most significant increase for both EGCG and EGCG-S (Figure 3(b)).

\subsection{EGCG and EGCG-S Showed Synergy with Antibiotics in Four GRAM-NEGATIVE BACTERIA}

Four Gram-negative bacteria, E. aerogenes, E. coli, P. aeruginosa and S. marcescens were used in this study. From the ZOI study, E. aerogenes was very resistant to most of the antibiotic treatments, with resistance to 9 (AM10, B10, CF30, D30, E15, P10, PB300, RA5, and TE30) antibiotics; intermediate to S10; and susceptible to only C30 and GM10. In the presence of EGCG, 4 (AM10, B10, PB300, and RA5) out of 9 antibiotics changed categories from resistant to susceptible, and 2 (E15 and P10) antibiotics changed from resistant to intermediate. It also changed S10 from intermediate to susceptible. EGCG was not able to convert CF30, D30 and TE30, thus E. aerogenes is still resistant to these three antibiotics. In the presence of EGCG-S, it converted PB300 from resistant to susceptible, 6 (AM10, B10, D30, P10, RA5 and TE30) antibiotics from resistant to intermediate, and S10 from intermediate to susceptible. Although the ZOI increased, EGCG-S did not convert susceptibility category on CF30 or E15 (Figure $4(\mathrm{a}))$.

EGCG and EGCG-S increased the antibiotic efficacy ranging from $19.32 \%$ to 
$144.44 \%$ and $12.66 \%$ to $164.29 \%$ respectively (Figure $4(\mathrm{~b}))$. AM10 (144.44\%) had the most significant increase with EGCG converting the antibiotic profile

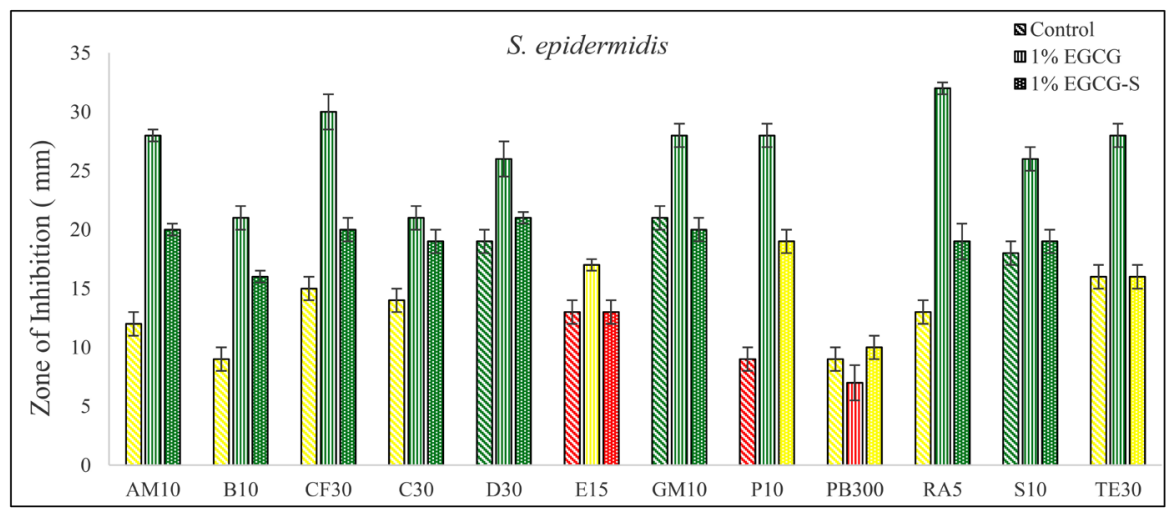

(a)

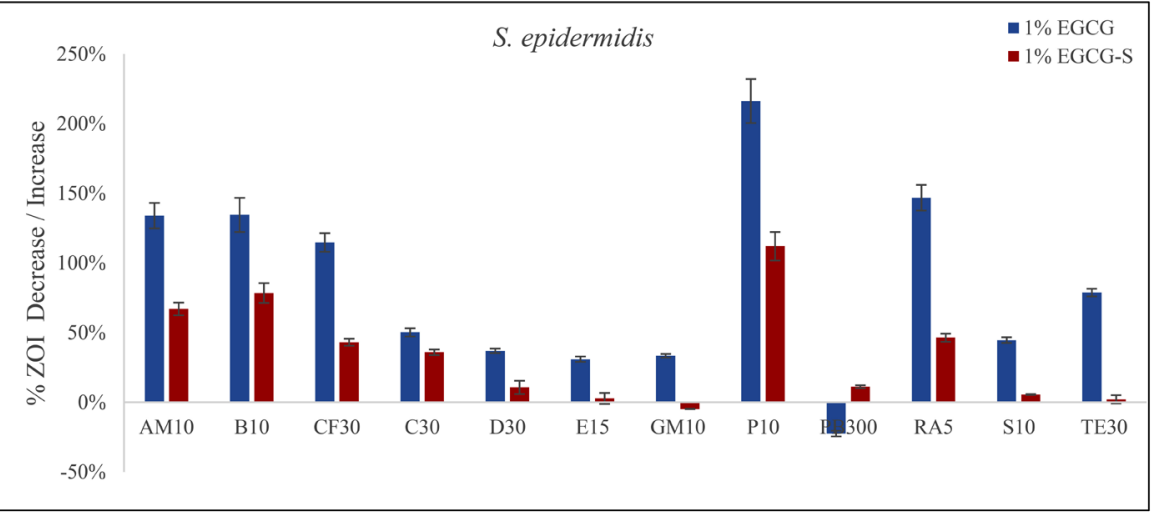

(b)

Figure 3. (a) The ZOI of $S$. epidermidis when treated with 1\% EGCG and 1\% EGCG-S in combination with antibiotics. The green color indicates susceptible, yellow indicates intermediate and red indicates resistant. The slanted stripe bar graph indicates antibiotic alone, the vertical stripe bar graph indicates antibiotic plus EGCG and the polka dot bar graph indicate antibiotic plus EGCG-S $(\mathrm{n}=3)$; (b) The percentage of ZOI increase/decrease of $S$. epidermidis when treated with 1\% EGCG and 1\% EGCG-S in combination with antibiotics. The blue bar represent antibiotic plus EGCG, and the red bar represent antibiotic plus EGCG-S $(n=3)$.

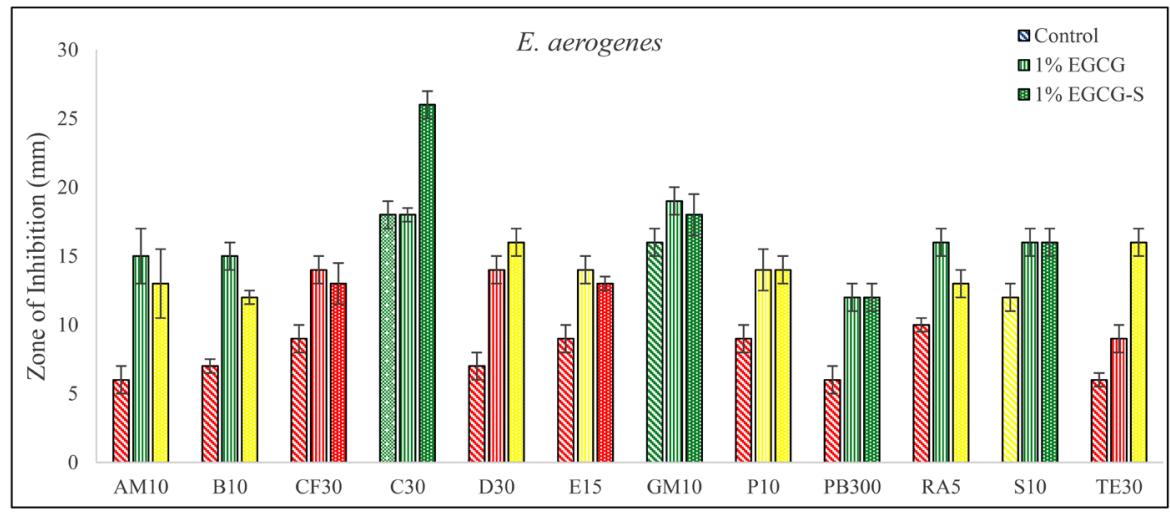

(a) 


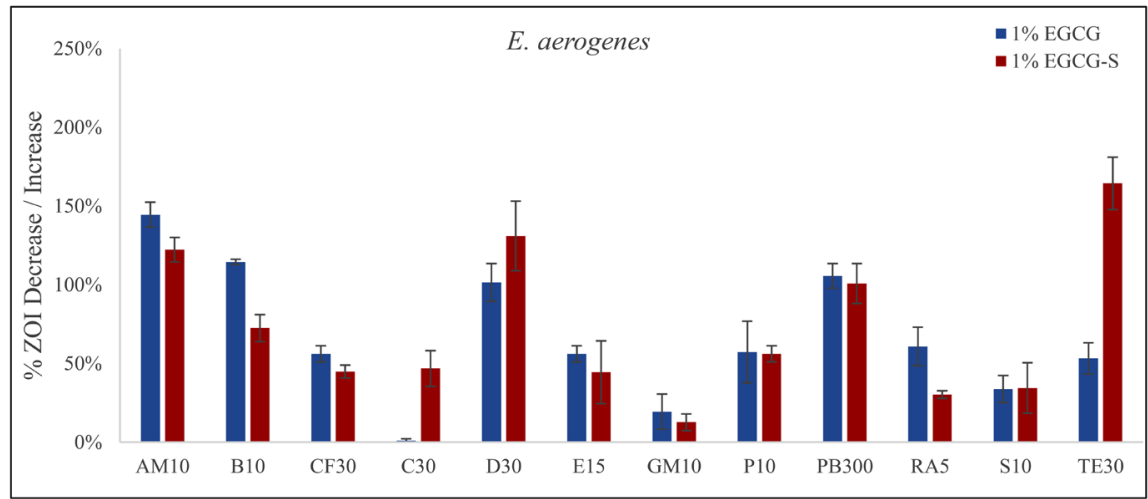

(b)

Figure 4. (a) The ZOI of E. aerogenes when treated with 1\% EGCG and 1\% EGCG-S in combination with various antibiotics. The green color indicates susceptible, yellow indicates intermediate and red indicates resistant. The slanted stripe bar graph indicates antibiotic alone, the vertical stripe bar graph indicates antibiotic plus EGCG and the polka dot bar graph indicate antibiotic plus EGCG-S $(n=3)$; (b) The percentage of ZOI increase/decrease of E. aerogenes when treated with 1\% EGCG and 1\% EGCG-S in combination with antibiotics. The blue bar represents antibiotic plus EGCG, and the red bar represents antibiotic plus EGCG-S $(n=3)$.

from resistant to susceptible while TE30 (164.29\%) had the most significant increase with EGCG-S converting the antibiotic profile from resistant to intermediate. The t-test statistical analysis was carried out and the p-value for both EGCG and EGCG-S were less than 0.01. Thus, these polyphenols have a synergistic effect on these antibiotics.

The ZOI study of $E$. coli indicates resistance to 5 (AM10, B10, D30, P10 and S10) of 12 antibiotics, intermediate to 4 (CF30, E15, RA5 and TE30) of 12 antibiotics, and susceptible to 3 (C30, GM10, and PB300) of 12 antibiotics. EGCG converted 4 (AM10, B10, D30 and S10) antibiotics from resistant to susceptible, 1 (P10) antibiotic from resistant to intermediate, and 2 (RA5 and TE30) antibiotics from intermediate to susceptible. EGCG-S was more effective than EGCG with a conversion of all initially resistant antibiotics to susceptible (AM10, B10, D30, and S10) except for P10 which was only converted to intermediate and all initially intermediate antibiotics to susceptible (CF30, E15, RA5, and TE30) (Figure 5(a)). The results suggested that EGCG-S is a much stronger synergistic anti-E. coli agent than EGCG.

EGCG and EGCG-S increased the antibiotic efficacy ranging from $10.93 \%$ to $212.04 \%$ and $2.10 \%$ to $183.33 \%$ respectively. EGCG had the highest efficacy for S10 (212.04\%) with a conversion from intermediate to susceptible, while EGCG-S had the highest efficacy for P10 (183.33\%) with a conversion from resistant to intermediate (Figure 5(b)). The t-test analysis resulted in a p-value of less than 0.05 for both EGCG and EGCG-S indicating a synergistic effect of both compounds to many antibiotics.

The results from the study of $P$. aeruginosa indicates that it is resistant to 6 (AM10, D30, E15, P10, RA5 and TE30) of 12 antibiotics. It is intermediate to 3 
(CF30, GM10 and S10) antibiotics and susceptible to 3 (B10, C30, PB300) antibiotics. Addition of EGCG did not convert any of the antibiotics from resistant to susceptible, but four of them were converted to intermediate susceptibility (AM10, D30, E15, and P10). EGCG also converted all the intermediate susceptibility to susceptible (CF30, GM10, and S10). Addition of EGCG-S has a more positive impact on the antibiotics; it converted E15 from resistant to susceptible and 5 (AM10, D30, P10, RA5 and TE30) antibiotics from resistant to intermediate. It also converted all the intermediate to susceptible. This result also illustrated that EGCG-S is more potent synergistic agent with antibiotics than EGCG (Figure 6(a)).

EGCG and EGCG-S increased the antibiotic efficacy from $7.17 \%$ to $115.87 \%$ and $7.17 \%$ to $176.19 \%$ respectively. EGCG had the most significant increase for E15 (144.4\%) but only converted the antibiotic resistant to intermediate. EGCG-S had the most\% of increase for E15 (176.19\%) and converted it from

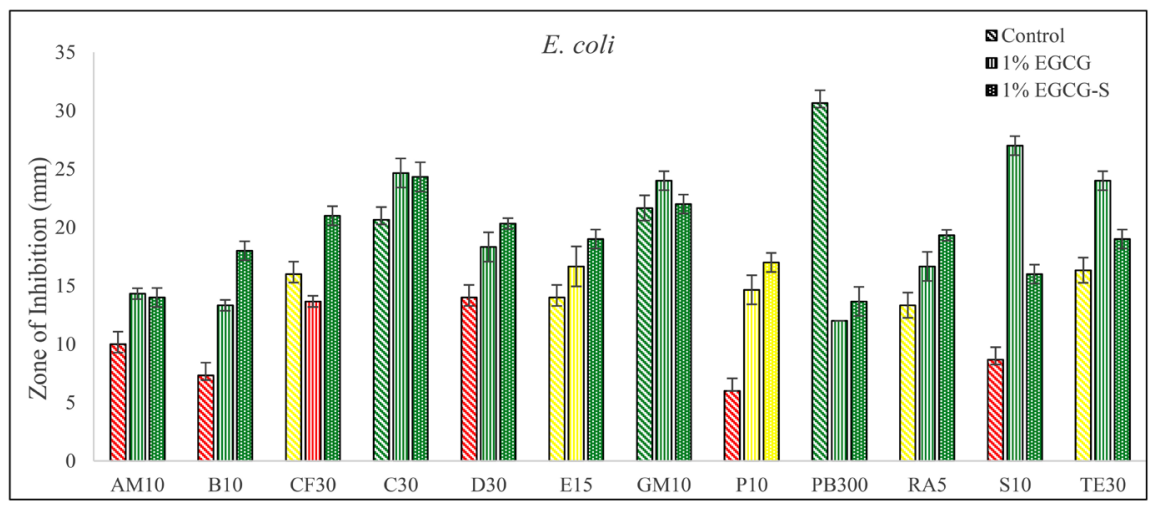

(a)

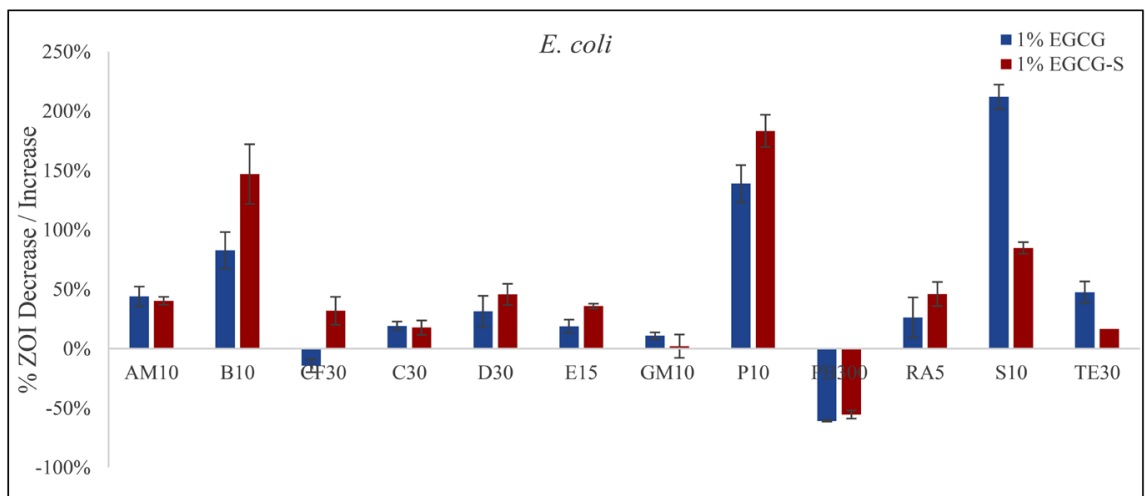

(b)

Figure 5. (a) The ZOI of E. coli when treated with 1\% EGCG and 1\% EGCG-S in combination with antibiotics. The green color indicates susceptible, yellow indicates intermediate and red indicates resistant. The slanted stripe bar graph indicates antibiotic alone, the vertical stripe bar graph indicates antibiotic plus EGCG and the polka dot bar graph indicate antibiotic plus EGCG-S; (b) The percentage of ZOI increase/decrease of E. coli when treated with 1\% EGCG and 1\% EGCG-S in combination with antibiotics. The blue bar represents antibiotic plus EGCG, and the red bar represents antibiotic plus EGCG-S $(\mathrm{n}=3)$. 


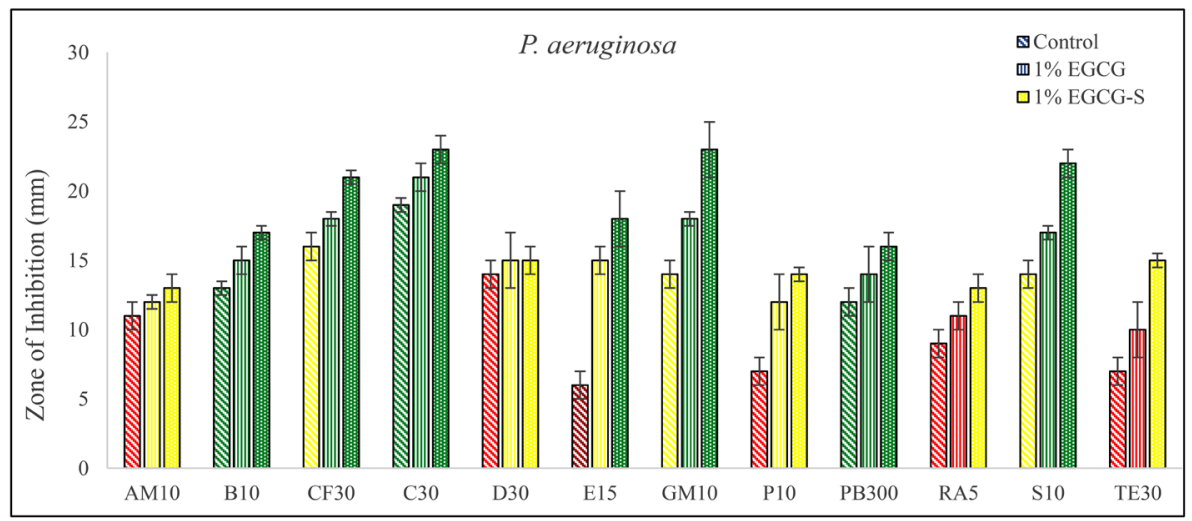

(a)

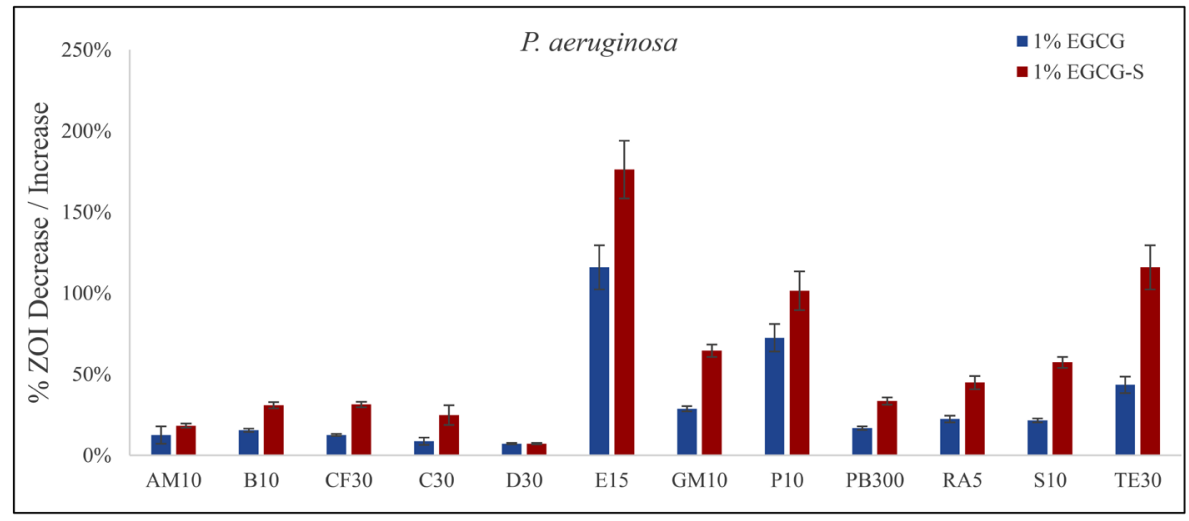

(b)

Figure 6. (a) The ZOI of $P$. aeruginosa when treated with 1\% EGCG and 1\% EGCG-S in combination with various antibiotics. The green color indicates susceptible, yellow indicates intermediate and red indicates resistant. The slanted stripe bar graph indicates antibiotic alone, the vertical stripe bar graph indicates antibiotic plus EGCG and the polka dot bar graph indicate antibiotic plus EGCG-S $(n=3)$; (b) The percentage of ZOI increase/decrease of $P$. aeruginosa when treated with $1 \%$ EGCG and $1 \%$ EGCG-S in combination with antibiotics. The blue bar represents antibiotic plus EGCG, and the red bar represents antibiotic plus EGCG-S $(n=3)$.

resistant to susceptible (Figure 6(b)). The t-test indicated a p-value of less than 0.05 for EGCG and less than 0.01 for EGCG-S demonstrating that the synergistic effect of both compounds are statistically significant.

The ZOI study for $S$. marcescens indicates higher tolerance for antibiotics, with resistance to 8 (AM10, B10, CF30, E15, P10, RA5, S10, TE30) of 12 antibiotics, intermediate resistance to $\mathrm{C} 30$ and D30, and sensitivity to only GM10 and PB300. In the presence of EGCG, C30 converted from intermediate to susceptible. EGCG-S was more effective than EGCG with the conversion of 3 (AM10, B10, and CF30) antibiotics from resistant to susceptible and 4 (P10, RA5, S10, and TE30) antibiotics from resistant to intermediate (Figure 7(a)). In addition, EGCG-S had greater ZOI percentage increases over a larger array of antibiotics ranging from $30.89 \%$ to $194.44 \%$, whereas EGCG-S had a ZOI percentage increase of $77.23 \%$ for C30 (Figure 7(b)). 


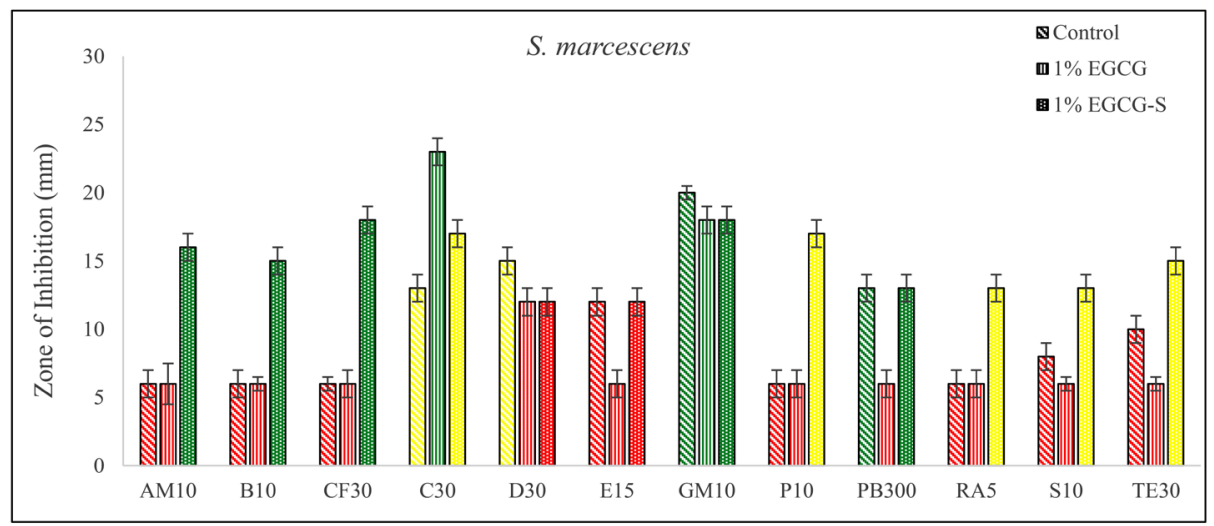

(a)

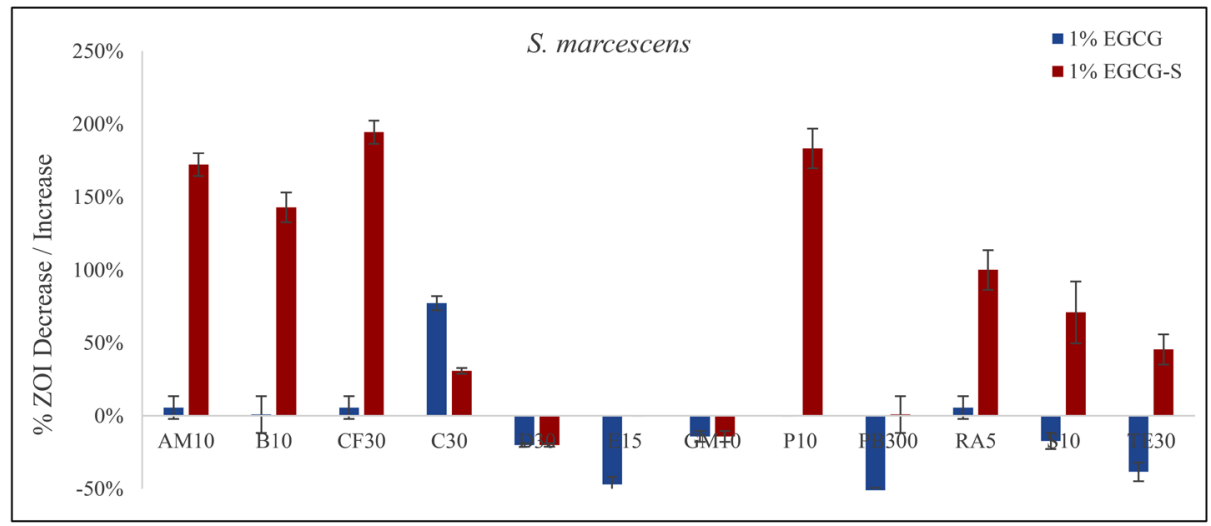

(b)

Figure 7. (a) The ZOI of $S$. marcescens when treated with 1\% EGCG and 1\% EGCG-S in combination with antibiotics. The green color indicates susceptible, yellow indicates intermediate and red indicates resistant. The slanted stripe bar graph indicates antibiotic alone, the vertical stripe bar graph indicates antibiotic plus EGCG and the polka dot bar graph indicate antibiotic plus EGCG-S ( $\mathrm{n}=3$ ); (b) The percentage of ZOI increase/decrease of $S$. marcescens when treated with 1\% EGCG and 1\% EGCG-S in combination with antibiotics. The blue bar represents antibiotic plus EGCG, and the red bar represents antibiotic plus EGCG-S $(n=3)$.

While EGCG had the greatest efficacy for C30 (77.23\%), this polyphenol only converted C30 from intermediate to susceptible, whereas EGCG-S had the greatest efficacy for CF30 (194.4\%) and was able to convert it from resistant to susceptible. The t-test statistical analysis demonstrated a p-value for EGCG-S of less than 0.01 indicating overall synergistic effects with antibiotics.

\subsection{EGCG and EGCG-S Showed Synergy with Antibiotics in Acid-Fast Bacteria}

The only acid-fast bacterium used for this study was $M$. smegmatis. The ZOI result of $M$. smegmatis indicated that the bacteria is resistant to 5 antibiotics (CF30, D30, PB300, S10, and TE30), intermediate to three antibiotics (AM10, E15, and P10), and susceptible to 4 antibiotics (B10, C30, GM10, and RA5). EGCG converted 3 antibiotics (D30, S10, and TE30) from resistant to suscepti- 
ble; PB300 from resistant to intermediate, and E15 from intermediate to susceptible. EGCG-S was more effective than EGCG in synergism with a conversion of 4 antibiotics (D30, PB300, S10, and TE30) from resistant to susceptible and one antibiotic (AM10) from intermediate to susceptible (Figure 8(a)).

EGCG and EGCG-S increased antibiotic efficacy ranging from $7 \%$ to $185.71 \%$ and $7 \%$ to $328.57 \%$ respectively. EGCG had the highest percentage increase of ZOI for S10 (185.71\%) with conversion from antibiotic resistance to susceptible. EGCG-S had the highest percentage increase for TE30 (328.57\%) with similar conversions of resistant to susceptible (Figure 8(b)).

The effect of crude green tea polyphenols (GTP) and crude lipophilic green tea polyphenols (LTP) on antibiotics has been reported [10]. The comparison of the synergistic effect of the pure EGCG and EGCG-S has been summarized in Table 1 . The results indicated that the green tea polyphenols have shifted the antimicrobial susceptibility category (R, I, and S) of the antibiotic in all the microorganisms. The EGCG and EGCG-S worked better than the crude extract (GTP and LTP). Both EGCG and EGCG-S worked comparably for Gram-positive. EGCG-S worked better than EGCG on Gram-negative bacteria and acid-fast bacteria. The 1\% EGCG-S enhanced seven antibiotics while EGCG only enhanced one antibiotic on in $S$. marcescens.

\section{Discussion}

We have previously reported that green tea polyphenols, both GTP and LTP, have synergistic antibacterial effects on some antibiotics against Gram+, Gramand acid-fast bacteria [10]. This study suggested that pure compounds of GTP and LTP, EGCG and EGCG-S, are the active ingredients for the synergy. Green tea, especially EGCG and its derivatives are widely popular for their several beneficial properties such as anti-inflammatory, anti-oxidant, anti-carcinogenic, anti-viral, anti-bacterial, anti-amyloidogenic, anti-biofilm and anticariogenic [20]-[25].

Despite the broad-spectrum effects, the antibacterial mechanisms of EGCG are still elusive. It has been reported that EGCG interacts with bacterial transcription potentially increasing cell permeability and thus facilities the entry of the antibiotics [23] [26] [27]. Some reported antibacterial mechanisms of EGCG include: 1) Induction of $\mathrm{H}_{2} \mathrm{O}_{2}$ resulting in disruption/lysis of outer membrane of Gram-negative bacteria [28] [29] [30]; 2) interference with glycocalyx and cell wall/cell membrane interactions [31] [32]; 3) inhibition of peptidoglycan synthesis [30] [33] [34] [35]; and 4) Bind to the fimbriae inhibiting bacterial adherence [36]. EGCG-S is a more stable derivative that can inhibit the production of bacteria secondary metabolites and thus induces endogenous oxidative stress and decreases biofilm formation [26]. Previous report also suggested that EGCG-S could damage the integrity of endospore coat [11]. The mechanisms of EGCG and EGCG-S may work synergistically with antibiotics to increase the susceptibility of antibiotic on antibiotic resistant bacteria. 
Table 1. Comparative analysis the synergistic effect of 1\% EGCG and 1\% EGCG-S.

\begin{tabular}{|c|c|c|}
\hline \multirow{2}{*}{ Bacteria } & \multicolumn{2}{|c|}{ Synergistic \% ZOI Increased } \\
\hline & $1 \%$ EGCG & $1 \%$ EGCG-S \\
\hline \multirow[t]{3}{*}{ B. megaterium } & Not significant & $\mathrm{B} 10(\mathrm{I} \rightarrow \mathrm{S}) 112.04 \%$ \\
\hline & AM10 $(\mathrm{R} \rightarrow \mathrm{I}) 9.14 \%$ & $\mathrm{AM} 10(\mathrm{R} \rightarrow \mathrm{S}) 54.85 \%$ \\
\hline & CF30 (I $\rightarrow$ S) $23.58 \%$ & CF30 $(I \rightarrow S) 35.38 \%$ \\
\hline \multirow[t]{6}{*}{ S. aureus } & C30 $(I \rightarrow S) 29.83 \%$ & $\mathrm{C} 30(\mathrm{I} \rightarrow \mathrm{S}) 35.48 \%$ \\
\hline & $\mathrm{S} 10(\mathrm{I} \rightarrow \mathrm{S}) 34.21 \%$ & $\mathrm{~S} 10(\mathrm{I} \rightarrow \mathrm{S}) 42.54 \%$ \\
\hline & TE30 $(\mathrm{I} \rightarrow \mathrm{S}) 28.14 \%$ & TE30 $(\mathrm{I} \rightarrow \mathrm{S}) 44.65 \%$ \\
\hline & AM10 $(\mathrm{I} \rightarrow \mathrm{S})$ 133.95\% & \\
\hline & $\mathrm{B} 10(\mathrm{I} \rightarrow \mathrm{S}) 134.44 \%$ & AM10 $(\mathrm{I} \rightarrow \mathrm{S}) 66.98 \%$ \\
\hline & CF30 $(\mathrm{I} \rightarrow \mathrm{S}) 114.68 \%$ & $\mathrm{~B} 10(\mathrm{I} \rightarrow \mathrm{S}) 78.43 \%$ \\
\hline \multirow{5}{*}{ S. epidermidis } & $\mathrm{C} 30(\mathrm{I} \rightarrow \mathrm{S}) 50.17 \%$ & CF30 (I $\rightarrow S) 43 \%$ \\
\hline & E15 $(\mathrm{R} \rightarrow \mathrm{I}) 30.89 \%$ & $\mathrm{C} 30(\mathrm{I} \rightarrow \mathrm{S}) 35.84 \%$ \\
\hline & $\mathrm{P} 10(\mathrm{R} \rightarrow \mathrm{S}) 216.20 \%$ & $\mathrm{P} 10(\mathrm{R} \rightarrow \mathrm{I}) 112.04 \%$ \\
\hline & RA5 $(\mathrm{I} \rightarrow \mathrm{S}) 146.73 \%$ & RA5 $(\mathrm{I} \rightarrow \mathrm{S}) 46.34 \%$ \\
\hline & TE30 $(\mathrm{I} \rightarrow \mathrm{S}) 78.75 \%$ & \\
\hline \multirow{8}{*}{ E. aerogenes } & AM10 $(R \rightarrow S) 144.44 \%$ & AM10 $(\mathrm{R} \rightarrow \mathrm{I}) 122.22 \%$ \\
\hline & $\mathrm{B} 10(\mathrm{R} \rightarrow \mathrm{S}) 114.48 \%$ & $\mathrm{~B} 10(\mathrm{R} \rightarrow \mathrm{I}) 72.42 \%$ \\
\hline & E15 $(\mathrm{R} \rightarrow \mathrm{I}) 18.78 \%$ & $\mathrm{D} 30(\mathrm{R} \rightarrow \mathrm{I}) 45.73 \%$ \\
\hline & $\mathrm{P} 10(\mathrm{R} \rightarrow \mathrm{I}) 57.22 \%$ & P10 (R $\rightarrow \mathrm{I}) 56.02 \%$ \\
\hline & $\mathrm{PB} 300(\mathrm{R} \rightarrow \mathrm{S}) 105.56 \%$ & PB300 $(\mathrm{R} \rightarrow \mathrm{S})$ 100.79\% \\
\hline & RA5 $(R \rightarrow S) 60.77 \%$ & RA5 (R $\rightarrow \mathrm{I}) 30.20 \%$ \\
\hline & $S 10(I \rightarrow S) 33.74 \%$ & $\mathrm{~S} 10(\mathrm{I} \rightarrow \mathrm{S}) 34.42 \%$ \\
\hline & $\mathrm{S} 10(\mathrm{I} \rightarrow \mathrm{S}) 33.74 \%$ & TE30 $(\mathrm{R} \rightarrow \mathrm{I}) 164.29 \%$ \\
\hline \multirow{9}{*}{ E. coli } & & $\mathrm{AM} 10(\mathrm{R} \rightarrow \mathrm{S})$ 40.27\% \\
\hline & AM10 $(\mathrm{R} \rightarrow \mathrm{S})$ 43.97\% & $\mathrm{B} 10(\mathrm{R} \rightarrow \mathrm{S}) 147.02 \%$ \\
\hline & $\mathrm{B} 10(\mathrm{R} \rightarrow \mathrm{S}) 82.74 \%$ & CF30 $(I \rightarrow S) 31.85 \%$ \\
\hline & $\mathrm{D} 30(\mathrm{R} \rightarrow \mathrm{S}) 22.03 \%$ & $\mathrm{D} 30(\mathrm{R} \rightarrow \mathrm{S}) 45.73 \%$ \\
\hline & $\mathrm{P} 10(\mathrm{R} \rightarrow \mathrm{I}) 138.89 \%$ & $\mathrm{E} 15(\mathrm{I} \rightarrow \mathrm{S}) 35.84 \%$ \\
\hline & RA5 $(\mathrm{I} \rightarrow \mathrm{S}) 26.24 \%$ & $\mathrm{P} 10(\mathrm{R} \rightarrow \mathrm{I}) 183.33 \%$ \\
\hline & $\mathrm{S} 10(\mathrm{R} \rightarrow \mathrm{S}) 212.04 \%$ & RA5 $(\mathrm{I} \rightarrow \mathrm{S})$ 45.94\% \\
\hline & $\mathrm{TE} 30(\mathrm{I} \rightarrow \mathrm{S}) 47.55 \%$ & $\mathrm{~S} 10(\mathrm{R} \rightarrow \mathrm{S}) 84.72 \%$ \\
\hline & & TE30 $(I \rightarrow S) 16.76 \%$ \\
\hline \multirow{9}{*}{$P$. aeruginosa } & & AM10 $(\mathrm{R} \rightarrow \mathrm{I}) 18.28 \%$ \\
\hline & AM10 $(\mathrm{R} \rightarrow \mathrm{I}) 12.47 \%$ & CF30 $(I \rightarrow S) 31.33 \%$ \\
\hline & CF30 $(I \rightarrow S) 12.53 \%$ & D30 (R $\rightarrow \mathrm{I}) 7.17 \%$ \\
\hline & $\mathrm{D} 30(\mathrm{R} \rightarrow \mathrm{I}) 7.17 \%$ & E15 $(R \rightarrow S)$ 176.19\% \\
\hline & E15 $(\mathrm{R} \rightarrow \mathrm{I}) 115.87 \%$ & GM10 $(\mathrm{I} \rightarrow \mathrm{S}) 64.51 \%$ \\
\hline & GM10 $(\mathrm{I} \rightarrow \mathrm{S}) 28.67 \%$ & $\mathrm{P} 10(\mathrm{R} \rightarrow \mathrm{I})$ 101.39\% \\
\hline & $\mathrm{P} 10(\mathrm{R} \rightarrow \mathrm{I}) 72.42 \%$ & RA5 (R $\rightarrow \mathrm{I}) 44.81 \%$ \\
\hline & $\mathrm{S} 10(\mathrm{I} \rightarrow \mathrm{S}) 21.50 \%$ & $\mathrm{~S} 10(\mathrm{I} \rightarrow \mathrm{S}) 57.34 \%$ \\
\hline & & TE30 $(\mathrm{R} \rightarrow \mathrm{I}) \quad 115.87 \%$ \\
\hline \multirow{7}{*}{ S. marcescens } & & AM10 $(R \rightarrow S)$ 172.22\% \\
\hline & & $\mathrm{B} 10(\mathrm{R} \rightarrow \mathrm{S}) 142.86 \%$ \\
\hline & & CF30 $(R \rightarrow S)$ 194.44\% \\
\hline & $\mathrm{C} 30(\mathrm{I} \rightarrow \mathrm{S})$ 77.23\% & $\mathrm{P} 10(\mathrm{R} \rightarrow \mathrm{I}) \quad 183.33 \%$ \\
\hline & & RA5 $(\mathrm{R} \rightarrow \mathrm{I}) 100 \%$ \\
\hline & & $\mathrm{S} 10(\mathrm{R} \rightarrow \mathrm{I}) 70.83 \%$ \\
\hline & & TE30 $(\mathrm{R} \rightarrow \mathrm{I}) 45.45 \%$ \\
\hline \multirow{5}{*}{ M. smegmatis } & $\mathrm{D} 30(\mathrm{R} \rightarrow \mathrm{S}) 110 \%$ & AM10 $(\mathrm{I} \rightarrow \mathrm{S})$ 41.67\% \\
\hline & E15 $(I \rightarrow S) 33.33 \%$ & $\mathrm{D} 30(\mathrm{R} \rightarrow \mathrm{S}) 250 \%$ \\
\hline & PB300 (R $\rightarrow$ I) $12.50 \%$ & PB300 $(\mathrm{R} \rightarrow \mathrm{S}) 137.50 \%$ \\
\hline & $\mathrm{S} 10(\mathrm{R} \rightarrow \mathrm{S}) 185.71 \%$ & $\mathrm{~S} 10(\mathrm{R} \rightarrow \mathrm{S}) 285.71 \%$ \\
\hline & TE30 $(\mathrm{R} \rightarrow \mathrm{S})$ 171.43\% & TE30 $(\mathrm{R} \rightarrow \mathrm{S})$ 328.57\% \\
\hline
\end{tabular}




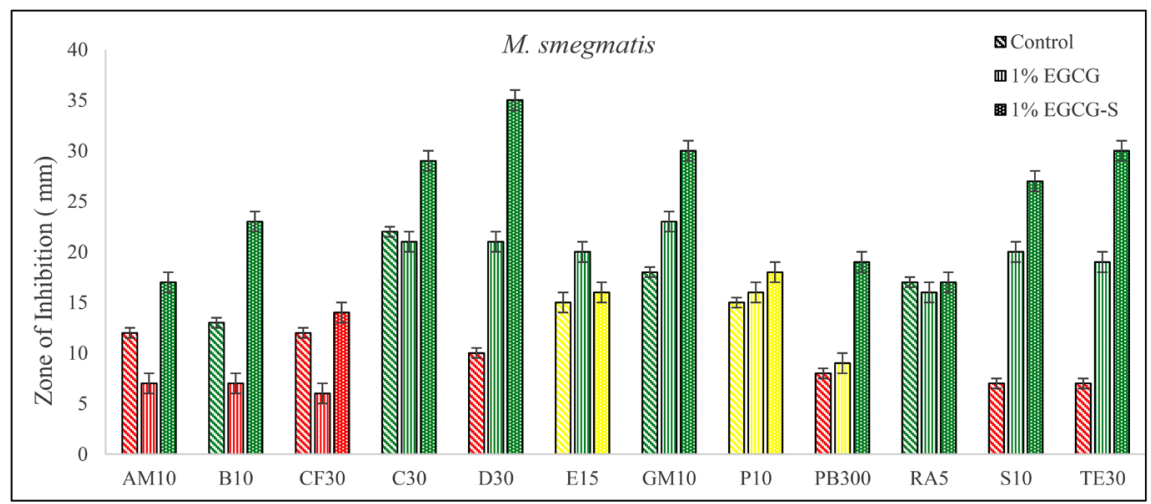

(a)

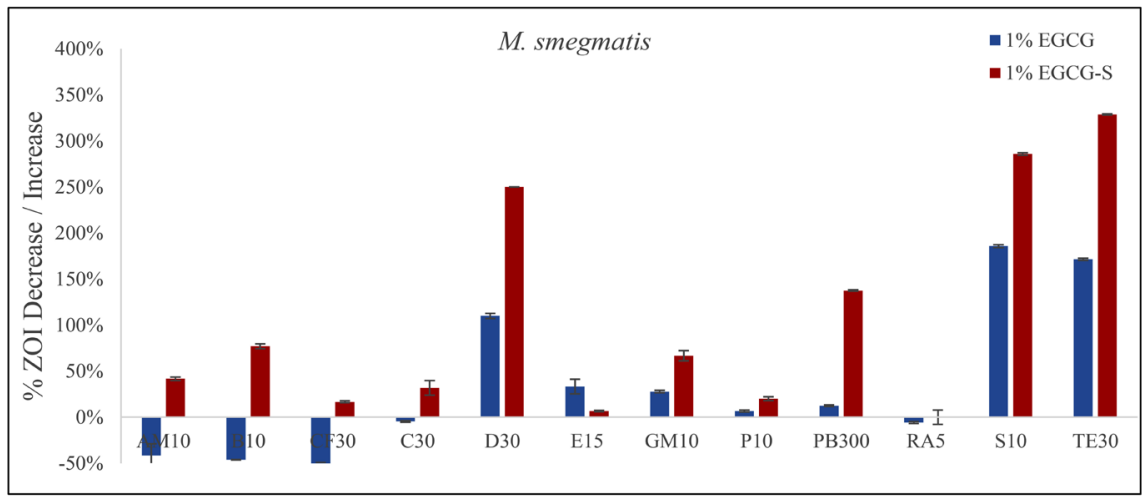

(b)

Figure 8. (a) The ZOI of $M$. smegmatis when treated with $1 \%$ EGCG and $1 \%$ EGCG-S in combination with antibiotics. The green color indicates susceptible, yellow indicates intermediate and red indicates resistant. The slanted stripe bar graph indicates antibiotic alone, the vertical stripe bar graph indicates antibiotic plus EGCG and the polka dot bar graph indicate antibiotic plus EGCG-S ( $=3$ ); (b) The percentage of ZOI increase/decrease of $S$. marcescens when treated with 1\% EGCG and 1\% EGCG-S in combination with antibiotics. The blue bar represents antibiotic plus EGCG, and the red bar represents antibiotic plus EGCG-S $(\mathrm{n}=3)$.

This study indicated that both EGCG and EGCG-S work synergistically with some antibiotics while EGCG-S possesses higher efficacy than EGCG. When combined with antibiotics, EGCG-S has particularly high potency on Gram-negative bacteria including $E$. aerogenes, $E$. coli, $P$. aeruginosa and $S$. marcescens. Usually the antibiotics are less effective on Gram-negative bacteria due to their outer membranes. Furthermore, both EGCG and EGCG-S enhance anti-mycobaterial activity significantly when combined with antibiotics. Overall, this study highlights that EGCG-S may serve as a promising synergistic agent with antibiotic.

\section{Conclusions}

This study looked into the potential use of green tea polyphenols EGCG and EGCG-S in combating the antibiotics resistant problem. This study used the Kirby-Bauer Assay to evaluate the effect of both EGCG and EGCG-S in combination with 12 antibiotics. 
For Gram-positive bacteria, the antibacterial effect of EGCG was comparable to EGCG-S as seen in Table 1. The result showed that EGCG was able to convert categories for 13 antibiotics while EGCG-S was able to convert categories for 12 antibiotics in $S$. aureus and $S$. epidermidis. For Gram-negative and acid-fast bacteria, EGCG is able to convert 27 antibiotics categories while EGCG-S is able to convert 38 antibiotics categories. This overall shows that EGCG-S works better in Gram-negative and Acid-Fast bacteria. Similar to Gram-positive bacteria, EGCG and EGCG-S worked better when compared to LTP and GTP [10]. S. marcescens for example, GTP and LTP were able to covert categories for 4 antibiotics [10] while EGCG and EGCG-S were able to convert categories for 8 antibiotics. It was important to note that EGCG-S increased the antibacterial activity up to $328.57 \%$ and converted the antibiotic effectiveness from resistance to susceptible category when combined with TE30. In summary, this study demonstrated that EGCG-S could serve as a synergistic antibacterial agent with antibiotics to combat the antibiotic problem.

\section{Acknowledgements}

This work was supported by Seton Hall University (SHU) Department of Biological Sciences Graduate Teaching Assistantship to A.Y.; Novartis Graduate Scholarship to U.H.; SHU Department of Biological Sciences Annual Research Fund and William and Doreen Wong Foundation to T.C.; and Montclair State University (MSU) Faculty Scholarship Program (FSP) to L.H.L.

\section{Conflicts of Interest}

The authors declare no conflicts of interest regarding the publication of this paper.

\section{References}

[1] Christou, A., Aguera, A., Bayona, J.M., Cytryn, E., Fotopoulos, V., Lambropoulou, D., Manaia, C.M., Michael, C., Revitt, M., Schroder, P. and Fatta-Kassinos, D. (2017) The Potential Implications of Reclaimed Wastewater Reuse for Irrigation on the Agricultural Environment: The Knowns and Unknowns of the Fate of Antibiotics and Antibiotic Resistant Bacteria and Resistance Genes-A Review. Water Research, 123, 448-467. https://doi.org/10.1016/j.watres.2017.07.004

[2] Molstad, S., Lofmark, S., Carlin, K., Erntell, M., Aspevall, O., Blad, L., Hanberger, H., Hedin, K., Hellman, J., Norman, C., Skoog, G., Stalsby-Lundborg, C., Tegmark Wisell, K., Ahren, C. and Cars, O. (2017) Lessons Learnt during 20 Years of the Swedish Strategic Programme against Antibiotic Resistance. Bull World Health Organ, 95, 764-773. https://doi.org/10.2471/BLT.16.184374

[3] Nesme, J., Cecillon, S., Delmont, T.O., Monier, J.M., Vogel, T.M. and Simonet, P. (2014) Large-Scale Metagenomic-Based Study of Antibiotic Resistance in the Environment. Current Biology, 24, 1096-1100. https://doi.org/10.1016/j.cub.2014.03.036

[4] WHO (2017) Global Priority List of Antibiotic-Resistant Bacteria to Guide Research, Discovery, and Development of New Antibiotics. 348-365.

[5] Santajit, S. and Indrawattana, N. (2016) Mechanisms of Antimicrobial Resistance in 
ESKAPE Pathogens. BioMed Research International, 2016, Article ID: 2475067. https://doi.org/10.1155/2016/2475067

[6] Si, W., Gong, J., Tsao, R., Kalab, M., Yang, R. and Yin, Y. (2006) Bioassay-Guided Purification and Identification of Antimicrobial Components in Chinese Green Tea Extract. Journal of Chromatography A, 1125, 204-210. https://doi.org/10.1016/j.chroma.2006.05.061

[7] Du, W., Zhou, M., Liu, Z., Chen, Y. and Li, R. (2018) Inhibition Effects of Low Concentrations of Epigallocatechin Gallate on the Biofilm Formation and Hemolytic Activity of Listeria monocytogenes. Food Control, 85, 119-126. https://doi.org/10.1016/j.foodcont.2017.09.011

[8] Isaacs, C.E., Wen, G.Y., Xu, W., Jia, J.H., Rohan, L., Corbo, C., Di Maggio, V., Jenkins, E.C. and Hillier, S. (2008) Epigallocatechin Gallate Inactivates Clinical Isolates of Herpes Simplex Virus. Antimicrobial Agents and Chemotherapy, 52, 962-970. https://doi.org/10.1128/AAC.00825-07

[9] Paterson, I. and Anderson, E.A. (2005) Chemistry. The Renaissance of Natural Products as Drug Candidates. Science, 310, 451-453.

https://doi.org/10.1126/science.1116364

[10] Haghjoo, B., Lee, L.H., Habiba, U., Tahir, H., Olabi, M. and Chu, T. (2013) The Synergistic Effects of Green Tea Polyphenols and Antibiotics against Potential Pathogens. Advances in Bioscience and Biotechnology, 4, 959-967. https://doi.org/10.4236/abb.2013.411127

[11] Ali, B., Lee, L.H., Laskar, N., Shaikh, N., Tahir, H., Hsu, S.D., Newby Jr., R., Valsechi-Diaz, J. and Chu, T. (2017) Modified Green Tea Polyphenols, EGCG-S and LTP, Inhibit Endospore in Three Bacillus spp. Advances in Microbiology, 7, 175-187. https://doi.org/10.4236/aim.2017.73014

[12] Chen, P., Dickinson, D. and Hsu, S.D. (2012) Lipid-Soluble Green Tea Polyphenols: Stabilized for Effective Formulation. Nova Science Publishers, Hauppauge.

[13] Chen, P., Tan, Y., Sun, D. and Zheng, X.M. (2003) A Novel Long-Chain Acyl-Derivative of Epigallocatechin-3-O-Gallate Prepared and Purified from Green Tea Polyphenols. Journal of Zhejiang University Science, 4, 714-718. https://doi.org/10.1631/jzus.2003.0714

[14] Doi, Y., Bonomo, R.A., Hooper, D.C., Kaye, K.S., Johnson, J.R., Clancy, C.J., Thaden, J.T., Stryjewski, M.E., van Duin, D. and Gram-Negative Committee of the Antibacterial Resistance Leadership Group (2017) Gram-Negative Bacterial Infections: Research Priorities, Accomplishments, and Future Directions of the Antibacterial Resistance Leadership Group. Clinical Infectious Diseases, 64, S30-S35. https://doi.org/10.1093/cid/ciw829

[15] Otero, F., Tamayo, M., Santiso, R., Gosalvez, J., Bou, G. and Fernandez, J.L. (2017) Rapid Assessment of Resistance to Antibiotic Inhibitors of Protein Synthesis in the Gram-Positive Pathogens, Enterococcus faecalis and Streptococcus pneumoniae, Based on Evaluation of the Lytic Response. Microbial Drug Resistance, 23, 267-271. https://doi.org/10.1089/mdr.2016.0091

[16] Cui, Y., Oh, Y.J., Lim, J., Youn, M., Lee, I., Pak, H.K., Park, W., Jo, W. and Park, S. (2012) AFM Study of the Differential Inhibitory Effects of the Green Tea Polyphenol (-)-epigallocatechin-3-gallate (EGCG) against Gram-Positive and Gram-Negative Bacteria. Food Microbiology, 29, 80-87. https://doi.org/10.1016/j.fm.2011.08.019

[17] Hindi, N.K.K., Abdul-Husin, I.F., Al-Mahdi, Z.K.A., Ewadh, R.M.J., Hossain, A.O., Kadhim, M.J., Alnasraui, A.H. and Al-Yaseri, A.A. (2017) Effectiveness of Aqueous Extract of Green, Black and Red Tea Leaves against Some Types of Gram Positive 
and Negative Bacteria. Research Journal of Pharmacy and Technology, 10, 1957-1962. https://doi.org/10.5958/0974-360X.2017.00343.2

[18] Nakasone, N., Higa, N., Toma, C., Ogura, Y., Suzuki, T. and Yamashiro, T. (2017) Epigallocatechin Gallate Inhibits the Type III Secretion System of Gram-Negative Enteropathogenic Bacteria under Model Conditions. FEMS Microbiology Letters, 364. https://doi.org/10.1093/femsle/fnx111

[19] CLSI (2009) Performance Standards for Antimicrobial Disk Susceptibility Test: Approved Standard. Clinical and Laboratory Standards, Institute, Wayne.

[20] Bieschke, J., Russ, J., Friedrich, R.P., Ehrnhoefer, D.E., Wobst, H., Neugebauer, K. and Wanker, E.E. (2010) EGCG Remodels Mature Alpha-Synuclein and Amyloid-Beta Fibrils and Reduces Cellular Toxicity. Proceedings of the National Academy of Sciences, 107, 7710-7715. https://doi.org/10.1073/pnas.0910723107

[21] Melok, A.L., Lee, L.H., Mohamed Yussof, S.A. and Chu, T. (2018) Green Tea Polyphenol Epigallocatechin-3-Gallate-Stearate Inhibits the Growth of Streptococcus mutans: A Promising New Approach in Caries Prevention. Dentistry Journal (Basel), 6. https://doi.org/10.3390/dj6030038

[22] Serafini, M., Del Rio, D., Yao, D.N., Bettuzzi, S. and Peluso, I. (2011) Health Benefits of Tea. CRC Press, Boca Raton. https://doi.org/10.1201/b10787-13

[23] Serra, D.O., Mika, F., Richter, A.M. and Hengge, R. (2016) The Green Tea Polyphenol EGCG Inhibits E. coli Biofilm Formation by Impairing Amyloid Curli Fibre Assembly and Down-Regulating the Biofilm Regulator CsgD via the Sigma(E)-Dependent sRNA RybB. Molecular Microbiology, 101, 136-151. https://doi.org/10.1111/mmi.13379

[24] Steinmann, J., Buer, J., Pietschmann, T. and Steinmann, E. (2013) Anti-Infective Properties of Epigallocatechin-3-Gallate (EGCG), a Component of Green Tea. British Journal of Pharmacology, 168, 1059-1073. https://doi.org/10.1111/bph.12009

[25] Xu, X., Zhou, X.D. and Wu, C.D. (2012) Tea Catechin Epigallocatechin Gallate Inhibits Streptococcus mutans Biofilm Formation by Suppressing gtf Genes. Archives of Oral Biology, 57, 678-683. https://doi.org/10.1016/j.archoralbio.2011.10.021

[26] Xiong, L.G., Chen, Y.J., Tong, J.W., Huang, J.A., Li, J., Gong, Y.S. and Liu, Z.H. (2017) Tea Polyphenol Epigallocatechin Gallate Inhibits Escherichia coli by Increasing Endogenous Oxidative Stress. Food Chemistry, 217, 196-204. https://doi.org/10.1016/j.foodchem.2016.08.098

[27] Zaveri, N.T. (2006) Green Tea and Its Polyphenolic Catechins: Medicinal Uses in Cancer and Noncancer Applications. Life Sciences, 78, 2073-2080.

https://doi.org/10.1016/j.lfs.2005.12.006

[28] Arakawa, H., Maeda, M., Okubo, S. and Shimamura, T. (2004) Role of Hydrogen Peroxide in Bactericidal Action of Catechin. Biological and Pharmaceutical Bulletin, 27, 277-281. https://doi.org/10.1248/bpb.27.277

[29] Betts, J.W. and Wareham, D.W. (2014) In Vitro Activity of Curcumin in Combination with Epigallocatechin Gallate (EGCG) versus Multidrug-Resistant Acinetobacter baumannii. BMC Microbiology, 14, 172. https://doi.org/10.1186/1471-2180-14-172

[30] Noormandi, A. and Dabaghzadeh, F. (2015) Effects of Green Tea on Escherichia coli as a Uropathogen. Journal of Traditional and Complementary Medicine, 5, 15-20. https://doi.org/10.1016/j.jtcme.2014.10.005

[31] Blanco, A.R., Sudano-Roccaro, A., Spoto, G.C., Nostro, A. and Rusciano, D. (2005) Epigallocatechin Gallate Inhibits Biofilm Formation by Ocular Staphylococcal Isolates. Antimicrobial Agents and Chemotherapy, 49, 4339-4343. https://doi.org/10.1128/AAC.49.10.4339-4343.2005 
[32] Ratnasooriya, W.D., Ratnasooriya, S.G. and Dissanayake, R. (2016) In Vitro Antibacterial Activity of Sri Lankan Orthodox Black Tea (Camellia sinensis L.) Belonging to Different Agro-Climatic Elevations. Journal of Coastal Life Medicine, 4, 623-627. https://doi.org/10.12980/jclm.4.2016J6-110

[33] Osterburg, A., Gardner, J., Hyon, S.H., Neely, A. and Babcock, G. (2009) Highly Antibiotic-Resistant Acinetobacter baumannii Clinical Isolates Are Killed by the Green Tea Polyphenol (-)-epigallocatechin-3-gallate (EGCG). Clinical Microbiology and Infection, 15, 341-346. https://doi.org/10.1111/j.1469-0691.2009.02710.x

[34] Yam, T.S., Hamilton-Miller, J.M. and Shah, S. (1998) The Effect of a Component of Tea (Camellia sinensis) on Methicillin Resistance, PBP2' Synthesis, and Beta-Lactamase Production in Staphylococcus aureus. Journal of Antimicrobial Chemotherapy, 42, 211-216. https://doi.org/10.1093/jac/42.2.211

[35] Zhao, W.H., Asano, N., Hu, Z.Q. and Shimamura, T. (2003) Restoration of Antibacterial Activity of Beta-Lactams by Epigallocatechin Gallate against Beta-Lactamase-Producing Species Depending on Location of Beta-Lactamase. Journal of Pharmacy and Pharmacology, 55, 735-740. https://doi.org/10.1211/002235703765951320

[36] Sakanaka, S., Aizawa, M., Kim, M. and Yamamoto, T. (1996) Inhibitory Effects of Green Tea Polyphenols on Growth and Cellular Adherence of an Oral Bacterium, Porphyromonas gingivalis. Bioscience, Biotechnology, and Biochemistry, 60, 745-749. https://doi.org/10.1271/bbb.60.745 